\title{
Variations
}

Variations

Revue internationale de théorie critique

$23 \mid 2020$

Pour une gauche érotique

\section{Le feu d'Eros.}

Pour Walter Benjamin - contre la mélancolie de gauche.

\section{Alexander Neumann}

\section{OpenEdition}

Journals

Édition électronique

URL : http://journals.openedition.org/variations/1818

DOI : 10.4000/variations. 1818

ISSN : 1968-3960

Éditeur

Les amis de Variations

Référence électronique

Alexander Neumann, «Le feu d'Eros. », Variations [En ligne], 23 | 2020, mis en ligne le 01 septembre 2020, consulté le 07 septembre 2020. URL : http://journals.openedition.org/variations/1818 ; DOI : https://doi.org/10.4000/variations. 1818

Ce document a été généré automatiquement le 7 septembre 2020

Les ami•e•s de Variations 


\section{Le feu d'Eros.}

Pour Walter Benjamin - contre la mélancolie de gauche.

\section{Alexander Neumann}

1 Certains pensent que le dieu Eros serait sorti d'un oeuf, d'autres qu'il nait de l'union d'Aphrodite et de Zeus, un écrivain grec l'a encore fantasmé en jeune beau, rien de tout cela n'est sûr. Peut-être qu'il brûlait comme le feu ou alors qu'il tirait ses flèches dans toutes les directions, comme un homme qui gicle, car les Grecs anciens l'ont érigé en stèle phallique aussi'. Quoi qu'il en soit, Sigmund Freud a bien saisi Eros comme une métaphore, comme le principe d'une puissance créatrice, agissante et pulsionnelle. Eros est le principe opposé à Thanatos, qui signale la mort, la peur, la dépression ou la mélancolie. Lors de la pandémie de 2020, il est apparu aux yeux de tous, de toutes, que la peur de mourir est un Thanatos qui pétrifie la pensée, empêche l'action collective, inhibe les désirs, appelle la dépression. La principale solution sanitaire que propose le pouvoir est de se voiler la face, pour se réfugier dans Facebook, d'interdire de se faire la bise et de faire la fête, de s'embrasser et de se toucher, de manifester et de se manifester. Tout doit passer par l'abstraction numérique, par les écrans qui font écran et qui détruisent l'expérience sensible, commune. Une perspective libératrice, critique, émancipatrice qui ne se laisse pas pétrifier a besoin de l'inspiration d'Eros, celle que Freud a cherché tout au long de sa vie. Ce regard permet de comprendre, comment Sigmund Freud a vaincu sa propre mélancolie en 1917, et pourquoi Walter Benjamin a rejeté avec la dernière énergie la mélancolie de gauche en 1931.

2 Pour bien saisir l'enjeu d'Eros et de Thanatos, qui ne se réduit pas à des colloques parisiens des sociétés de psychanalyse, il s'agit de comprendre le moment précis, où la pensée freudienne passe de la mélancolie à la puissance d'agir, à travers la révolution des conseils de 1917-18. La révolution s'ébroue d'abord en Russie, puis en Allemagne, enfin en Autriche-Hongrie et à Vienne, c'est elle qui met fin à la guerre et sa barbarie. Le penseur va alors passer de la peur de la mort au désir du changement. Pendant la première guerre mondiale, Freud est inquiet, abattu, sans doute déprimé, et littéralement inhibé. En 1915, il publie un essai sur l'état dépressif, le suicide et la mort : Deuil et mélancolie ${ }^{2}$. Ici, il montre que la dépression comporte toujours une envie subconsciente de se punir soi-même. Pendant ce temps, il s'échine de rédiger une série 
de sept autres essais qui devaient actualiser l'état des recherches et discussions internationales de la psychanalyse, et qui auraient pu représenter l'équivalent d'un livre. Alors qu'il avait complété une première version de ces textes, il décide de détruire les manuscrits, à l'été 1918, ce qui est une manière d'acter une impasse intellectuelle. Il n'arrivait pas à répondre de manière satisfaisante aux interpellations critiques, qui voulaient amoindrir la portée de sa découverte des pulsions d'origine sexuelle pouvant expliquer nombre de motivations sociales. Adler préfère limiter l'analyse à l'attraction du pouvoir, alors que Jung imagine un inconscient collectif - une théorisation qu'il va plus tard accorder avec l'idéologie raciste des nazis. En 1918, Freud constate que sa compréhension psychologique des comportements, en apparence irrationnels, a été confortée par l'expérience auto-destructrice de la guerre, tout en cherchant à prolonger sa théorie sexuelle qui fut publiée pour la première fois en 1914. Dès 1920 , il commence à réunir des matériaux et des notes pour saisir la dimension proprement collective des phénomènes, qu'il avait analysé sur la base de cas personnels dans sa pratique clinique. ${ }^{3}$ Désormais, il s'intéresse aux mouvements et organisations de masse. Vers la fin de la guerre, Freud avait déjà plaidé pour une psychothérapie populaire, lors du congrès de Budapest ${ }^{4}$, avant de se prononcer en faveur de cliniques prolétariennes gratuites. ${ }^{5}$ Une approche similaire sera pratiquée après la guerre par Wilhelm Reich dans le Ambulatorium de Vienne, que Freud soutient en pratique et en public. L'observation des névroses, la pratique clinique et démocratique pour les saisir et guérir, fait intégralement partie de l'élaboration intellectuelle des concepts freudiens. Ces réflexions nouvelles culminent chez Freud dans un livre qui relie les deux versants de la vie personnelle et sociale, sans les confondre : Analyse du moi et psychologie des masses ${ }^{6}$. Le geste fondateur de Freud, consistant à détruire ses manuscrits dominés par la peur, en 1918, cède alors la place à un livre d'un genre théorique nouveau, qui trouve ses sources et ressources dans la révolution des conseils. Sans exclure les mouvements syndicaux ou politiques, Freud y analyse en premier lieu les organisations de masse issues de l'empire défait : l'Eglise catholique et l'Armée.

La puissance révolutionnaire de Sigmund Freud reste complètement mésestimée sur le plan théorique, en particulier la forme qu'elle prend à travers son élaboration de la psychologie de masse. Pourtant, l'attitude très favorable de Freud envers la révolution des conseils est bien documentée, loin de la caricature d'une psychanalyse prétendument individualiste, bourgeoise et apolitique. En octobre 1917, il trouve "extraordinairement passionnant" les pourparlers de paix qui sont alors entamées par Trotsky, au nom du jeune gouvernement russe. Plus nettement encore, il juge : "Il est bon que la vielle époque meure, mais la nouvelle n'est pas née encore", en précisant qu'il n'allait pas verser "une larme sur cette Allemagne ou cette Autriche"7. L'année suivante la révolution des conseils y triomphe, avènement que Freud commente explicitement dans ses notes quotidiennes ${ }^{8}$. Il y écrit noir sur blanc : "Révolution à Vienne et Budapest" (30/10/1918); "Armistice avec l'Italie, la guerre est finie!" (3/11/18); "Prix Nobel, hors de question!" (4/11/18); "Révolution à Kiel!" (6/11/18); "République en Bavière!!" (8/11/18); "République à Berlin, Guillaume abdique?" (9/11/18); "Fin de la guerre" (11/9/18). Sa joie de voir le continent libéré des empires militaristes et morbides va de pair avec le soulagement d'apprendre la démobilisation de ses fils qui risquaient de mourir au front. Cette libération marque profondément la pensée et les écritures de Freud ${ }^{9}$.

4 La Théorie critique d'origine francfortoise va s'inscrire complètement dans cette impulsion créatrice, freudienne, révolutionnaire, ce qu'une partie de l'intelligentsia parisienne n'a jamais pu admettre, écartant d'un revers de main ce "freudo-marxisme" 
si troublant, si débordant. La filiation est particulièrement nette dans les études au sujet du fascisme (et de ses oppositions), depuis La psychologie de masse du fascisme de Reich (1933), De Caligari à Hitler de Kracauer (1947), Autorité et famille sous l'égide de Marcuse (suivi de Eros et civilisation), La personnalité autoritaire dirigée par Adorno en 1951 (dont l'introduction signale la fondation freudienne), jusqu'à un projet d'habilitation d'Adorno entièrement tourné vers la théorie sociale et psychanalytique inspirée de Freud (consultable aux Archives Adorno et Benjamin à Berlin). La liste complète des ouvrages et articles qui se réfère à cet héritage serait bien trop longue pour être égrenée ici. Lors du séminaire fondateur de l'Institut de Francfort de mai 1923, Lukacs évoque et nomme l'enjeu d'une analyse de la psychologie des masses, en discussion directe avec Karl Korsch ${ }^{10}$. Pour ce qui concerne Walter Benjamin, il rencontre Adorno et Kracauer pour la première fois à Francfort, la même année, pour discuter les thèses de Lukacs, puis les implications théoriques des mouvements révolutionnaires. Le rapport benjaminien à Freud passe en premier lieu par la révolution surréaliste, dont le manifeste fondateur, présenté par Breton, foisonne de références freudiennes, en particulier lors de ses séjours parisiens ${ }^{11}$. Sans surprise, les biographes ont saisi le pouvoir d'attraction de la ville Paris, une idée qui frise le cliché, mais qui s'incarne de manière sensible pour Benjamin. Lorsqu'il rencontre Colette de visu, à Paris, il la confronte avec la théoricienne de la révolution sexuelle, Alexandra Kollontaï, pour tester la position de son interlocutrice sur la banalisation sexuelle. Colette lui réplique alors avec aplomb: "On y a rien fait que l'amour"12. Les passages de Paris provoquent des illuminations benjaminiennes.

5 Pendant cette période, Adorno encourage Benjamin à se pencher sur les ressources freudiennes pour donner de la force à son Livre des passages (Paris, Capitale du 19ème siècle), dans une lettre de 1935 : Vous êtes ici, peut-être sans le savoir, en très profond accord avec Freud. La réponse de Benjamin sur ce sujet est explicite, lorsqu'il aborde la médiation entre psychologie et société : En effet, nous tirons là - et sans que j'en aie eu conscience auparavant avec cette formulation - sur une seule même corde (...) Je vais m'occuper de Freud prochainement ${ }^{13}$.

\section{Benjamin en quête d'Eros}

6 Au début des années 1930, Benjamin vit déjà entre Paris et Berlin, c'est durant cette période qu'il rédige une critique littéraire au sujet du poète Erich Kästner, en 1931, qui porte le titre : Mélancolie de gauche. L'auteur de ces lignes a souhaité rendre accessible le texte au public francophone, car il eut été difficile d'exposer toutes les distorsions qu'a subi ce thème benjaminien en France sans le rendre accessible dans la langue de Molière. Benjamin y définit la mélancolie de gauche ainsi: Ce genre de radicalisme de gauche correspond précisément à l'attitude qui ne se prolonge par aucune action politique. Il ne se situe pas à gauche d'un courant précis, mais se place à la gauche du possible en général. Puisque, dès le départ, il n'a d'autre objectif que jouir de sa propre existence, dans un repos qui se complait dans le refus ${ }^{14}$.

7 La poésie de Kästner est mélancolique, elle manque du ressort vif des écritures de Brecht, Breton ou Benjamin. Parmi les vers épinglés par la critique benjaminienne se trouve le titre évocateur "Suicide à la piscine publique": ${ }^{15}$

Tristement porté par les vertes vagues

une femme blonde plantée devant mon nez

Hélas, la mer n'offre pas de places vacantes 
et les gens nous bouchent toute visée

Une seule issue demeure ici: se noyer!

L'on se fait lourd comme une pierre

lentement l'on se laisse remplir d'eau

Sur le fond de la mer l'on est solitaire

Pour Benjamin, la mélancolie de gauche est la plus pure expression de l'opportunisme d'une couche d'intellectuels petit-bourgeois qui surfe sur les modes mondaines de la gauche, un groupe de parvenus dont la mélancolie est pétrie de cynisme, de conformisme, et qui singe les thèmes contre-culturels pour les mettre à son profit. Il enfonce le clou, sans modération aucune, à l'encontre de la lyre de Kästner, dans un verdict sans appel : La stupidité tourmentée: c'est le dernier résidu des métamorphoses de la mélancolie qui se jouent depuis deux mille ans. La critique benjaminienne semble dure, implacable, elle pourrait choquer, si l'histoire ne lui avait pas donnée entièrement raison. Car après la prise de pouvoir de Hitler, Kästner s'arrange aussitôt, il oublie tout radicalisme de gauche et accepte de produire, en 1934, un roman de divertissement, une production littéraire qui est parfaitement conforme aux attentes médiatiques du pouvoir en place (Drei Männer im Schnee). En 1942, il écrit le scénario pour le film Münchhausen sous le contrôle du Ministère de la propagande. La prise du pouvoir provoqua une prose du pouvoir. Kästner lui-même s'est chargé de confirmer l'accusation d'être un parvenu apolitique et cynique que Benjamin lui avait adressé. Il serait donc faux de croire qu'il s'agisse là d'une charge exagérée ou pamphlétaire, venue de Benjamin, qui se contente en réalité de dresser un constat objectif, constat qui se vérifie aussitôt dans la collaboration avec le régime nazi de Kästner, ce mélancolique de gauche devenu un dépressif divertissant. La contre-proposition de Benjamin est bien connue. Il jette ses thèses sur le concept d'histoire à la face de l'adversaire fasciste, après avoir soutenu avec force Bertold Brecht, le mouvement surréaliste, la Théorie critique. Les thèses sur le concept d'histoire déploient une conception dialectique, opposée en tous points à l'ontologie, à la tradition et en ces sens à la commémoration rituelle (des guerres, des morts). L'approche de Benjamin est celle du souvenir actif, à travers l'expérience vivante du présent, une conception qui est largement inspirée de la pensée de Freud. Dans la théorie et la pratique psychanalytique, le souvenir favorise l'accès à des questions et problèmes du présent, et ce travail permet éventuellement de surmonter la mélancolie, la dépression ou l'impuissance.

9 De la même manière que la question de la mélancolie chez Freud - qui sera dépassée grâce à la révolution des conseils - fait encore parfois l'objet de schématisations désuètes qui veulent cantonner les ressorts de la psychanalyse à une forme individualiste et bourgeoise, la prise de position de Walter Benjamin fait, elle aussi, l'objet d'interprétations erronées ou abusives. Le texte et le thème benjaminien de la mélancolie de gauche a manifestement subi ce sort. Pour saisir l'ampleur des abus et biais pamphlétaires, il est utile de revenir d'abord sur les attaques que Benjamin a subi de son vivant, et qui poursuivent leur oeuvre de nos jours.

\section{Détours, détournements et tourments soviétiques}

10 Une illustration caricaturale du problème, celui d'un recyclage d'attaques idéologiques anciennes, est aujourd'hui livrée par Razmig Keucheyan. L'auteur répète, en effet, une vielle pièce qui postule, inlassablement, une proximité de l'Ecole de Francfort et de Benjamin avec des penseurs nazis: Walter Benjamin avait subi l'influence de Schmitt. (...) 
Une proximité peut être constaté entre Schmitt les penseurs de l'Ecole de Francfort. Celle ci résulte de la similitude des expériences historiques qu'ils ont vécues, à commencer par celle de la république de Weimar dont ils sont issues ${ }^{16}$. Cette dénonciation calomnieuse d'un Benjamin proche des penseurs du national-socialisme est, hélas, une émanation directe de la propagande stalinienne, qui a voulu présenter Benjamin comme une sorte de hitlerotrotskyste (terme issu de l'arsenal stalinien destiné à disqualifier toutes les critiques de gauche), lui qui avait parfaitement compris les dégâts autoritaires de l'Union soviétique, dès 1927. Plus précisément, il fut diffamé par la presse stalinienne comme un ami des Nazis, de Carl Schmitt ou de son colistier nazi Martin Heidegger en particulier (tous deux jurent allégeance à Hitler en 1933 et restent membres du parti nazi jusqu'en 1945). Pareille charge pamphlétaire à l'encontre de Benjamin, qui est un intellectuel antifasciste de la première heure, se trouve par exemple publiée dans la revue pro-soviétique et stalinienne allemande, Internationale Literatur, en $1938^{17}$. Le procédé stalinien est particulièrement cynique, au moment où Staline négocie un accord militaire avec Hitler - le pacte Molotow-Ribbentropp - qui entre en vigueur en 1939. Aujourd'hui, ces constructions idéologiques semblent désuètes, au vu des procès de Nuremberg qui jugea les véritables responsables Nazis en 1946, puis de l'effondrement final de l'Union soviétique. La caricature est néanmoins entretenue, à la manière d'une légende urbaine qui croit savoir que l'épinard serait très riche en fer (erreur initialement provoquée par le déplacement fortuit d'une virgule dans un livre de médecine vers 1890$)^{18}$.

11 Pour sa part, Traverso reprend ce préjugé ancien qui voit en Adorno un intellectuel apolitique et pessimiste qui n'aurait pas cru à la révolution, car il n'aurait pas vécu la révolution russe. ${ }^{19} \mathrm{Ce}$ cliché est aussi repris par Keucheyan, qui recycle à son tour une prise de position de Perry Anderson de 1974, qui avait fait son mea culpa dans une postface deux ans plus tard, en 1976. Anderson constate que les conclusions de son essai ont pu inviter à une lecture activiste, "scientifiquement insoutenable et politiquement irresponsable"20. Pareille position - insoutenable et irresponsable - inspire aujourd'hui certains auteurs postmarxistes dans leur opposition à la Théorie critique. Errare humanum est, perseverare diabolicum. Anderson avait mis en garde contre la "logique réductrice" de son propre essai, écrit à la hâte, le "ton apocalyptique", les difficultés "esquivées" ou volontairement ignorées, et surtout la "faiblesse centrale" de son approche. Celle d'avoir tenté d'esquisser une histoire du matérialisme dialectique, de facture marxienne, en omettant complètement de s'approprier l'histoire réelle des mouvements d'émancipation qui produisent les théories critiques, leur sens pratique et leur portée. Il est vrai qu'Adorno a en réalité vécu la révolution des conseils allemande de 1918 qui suit la révolution russe - tout comme Benjamin - et qui constitue l'expérience historique fondatrice de la Théorie critique francfortoise.

12 Ajoutons que le stéréotype le plus répandu, celui d'un Adorno pessimiste (reproche étendu à Benjamin) répond lui aussi à une commande directe du régime stalinien, qui l'a fait écrire à Lukacs et d'autres quand ils étaient pris et surveillé à l'Hôtel Lux à Moscou durant la guerre mondiale. En 1933, Lukacs fuyant les Nazis, doit se repentir devant les comités soviétiques et faire allégeance à Staline, notamment à travers un pamphlet dirigé contre les écrivains allemands qui s'opposent à Hitler sans pour autant rallier Staline. Thomas Mann se trouve ainsi dénoncé, sous la plume de Lukacs, comme étant un intellectuel éthéré, défaitiste et pessimiste, qui s'enivre de l'abime qui s'ouvre devant lui, dans un Grand Hôtel l'Abysse. ${ }^{21}$ Lukacs ne reprend ce titre et cette veine 
pamphlétaire contre Adorno qu'en 1951 (lors d'une nouvelle préface allemande à sa théorie du roman), toujours sur le même thème du Grand Hôtel l'Abysse, pour y présenter le penseur francfortois comme un pessimiste ${ }^{22}$. Les Minima moralia d'Adorno, qui circulent la même année, comportent des remarques ironiques à l'égard des intellectuels staliniens: Les intellectuels oublient Proust dès que Staline tousse ${ }^{23}$. Dans la vision soviétique, être anti-stalinien c'est pessimiste, alors qu'être stalinien serait optimiste. Le fait que Lukacs soit poussé à adopter cette optique à partir de 1933 masque un moment tragique, puisque c'est l'optimisme aveugle de Staline - croyant à un renversement pro-soviétique facile du gouvernement de droite allemand - qui a favorisé la victoire de Hitler en 1933. Lukacs prête aux intellectuels allemands nonconformistes l'idée d'habiter un Grand Hôtel l'Abysse, au moment où il entre dans le Grand Hôtel Lux, qui est le lieu où Staline regroupe les exilés communistes pour les surveiller. Lukacs s'y tient au bord de l'abime, il risque de tomber dans le vide et de mourir à chaque instant, et il frôle effectivement la mort lors d'un emprisonnement par la police politique soviétique (NKWD) en 1941. Le trait d'esprit de Lukacs du Grand Hôtel l'Abysse pourrait être interprété comme une projection qui exprime son propre malheur, sa détresse d'être poussé à trahir la Théorie critique dont il fut un animateur au départ, lors du premier séminaire de l'Institut de Francfort de mai 1923. L'abysse est une image bien choisie pour signaler l'abime au bord duquel se loge la dépression.

Malheureusement, Benjamin n'a pas trouvé beaucoup de défenseurs de son vivant en France, ce qui explique sans doute les distorsions idéologiques énormes qui entourent sa pensée et ses écrits. Lors de son exil parisien, qui signale à quel point Benjamin est éloigné des théoriciens nazis, l'intellectuel internationaliste est handicapé par le fait que les revues littéraires les plus en vue refusent catégoriquement de publier le moindre article en français qu'il leur soumet, notamment la NRF édité par Gallimard. Il est éclairant de voir que la NRF a accepté, en revanche, un accord avec l'occupant nazi à Paris, accord qui a conditionné la parution de la revue à l'élimination de tous les membres juifs ou antifascistes de son comité de rédaction, en $1940^{24}$. Réduit au silence par des revues autant mondaines qu'opportunistes, vilipendé par les staliniens menteurs de tous les pays, Benjamin a eu le plus grand mal à faire reconnaitre ses idées et positions à Paris de son vivant. Aujourd'hui, il fait l'objet d'une mode qui assure sa visibilité et la diffusion large de ses écrits, mais le fait qu'il ne soit plus là pour les défendre autorise toute sorte d'intellectuels à parler en son nom, à tort et à travers.

Ces dernières années, l'édition et la presse parisienne ont repris le leitmotiv benjamin de la mélancolie de gauche. Ici, il ne s'agit plus seulement de recycler les préjugés de l'époque stalinienne ou soviétique, mais de reprendre les titres de Benjamin pour lui faire dire exactement l'inverse de ce qu'il a écrit. Benjamin n'est certainement pas le représentant d'une mélancolie de gauche, opportuniste, cynique et impuissante, celle qu'il a voulu dénoncer au contraire, avec beaucoup de vigueur, dans son article éponyme. Il est désormais facile de s'en convaincre, à la découverte de la traduction française du texte ${ }^{25}$. Si le stalinisme a voulu faire de Benjamin un ami des Nazis, certains essayistes ou journalistes voudraient aujourd'hui faire de Benjamin l'ami de Kästner, qui est en réalité au coeur de sa critique. Si la simple publication du texte benjaminien suffit à dissiper ce malentendu, il me semble intéressant de discuter plus en détail le cas de la distorsion mentale autour de la mélancolie de gauche, dans la mesure où cet exemple est symptomatique d'une série de confusions ou d'inversions intellectuelles qui font florès au sein de la galaxie postmarxiste. 


\section{Lutte des classes versus mélancolie de gauche}

Mélancolie de gauche est le titre d'un livre d'Enzo Traverso qui a eu un certain écho. ${ }^{26} \mathrm{Si}$ le titre reprend mot à mot celui de l'article de Benjamin, le contenu tente de contredire le grand modèle. Le livre semble vouloir anéantir toutes les positions, approches et conceptions de Benjamin, point par point, au delà même de la poésie pseudo-radicale d'un Kästner qui avait motivé la critique benjaminienne. Chez Benjamin, la mélancolie de gauche est une posture détestable, l'expression pure d'une petite bourgeoisie arriviste qui se dit de gauche, mais qui nie la réalité sociale de la lutte des classes. Chez Traverso, cette même expression mélancolique apparait soudainement comme une chose admirable, elle se trouve mise au service d'un enterrement de toutes les impulsions révolutionnaires que partagea Benjamin de son vivant. Chez Benjamin, la mélancolie de gauche désigne une impuissance politique, chez Traverso elle est acceptée comme une fatalité et comme un fardeau à porter. Chez Benjamin, elle se montre incapable de s'affranchir du conformisme et de la tradition, chez Traverso elle est positivement enrôlée dans la tradition. Le contre-sens est évident, tout comme l'impression que Traverso n'a pas lu le texte original de Benjamin, Mélancolie de gauche, d'autant qu'il n'en donne pas la source et qu'il ne le cite à aucun moment dans son livre de 230 pages. Le contre-sens s'y manifeste en gros comme en détail. Si Benjamin voit le Cuirassé Patiomkine d'Eisenstein comme une forme artistique qui échappe à l'idéologie soviétique dominante, Traverso veut en faire la pure illustration d'une tradition marxiste-soviétique. Ici, Traverso contredit Benjamin sans même le savoir, affirmant que les films d'Eisenstein participeraient d'une seule et même "tradition soviétique" qui mettrait en scène "l'histoire russe comme une ascension irrésistible vers la révolution"27. Benjamin, lui, avait pourtant présenté le Cuirassé Patiomkine (1925) comme un exemple de la potentialité nouvelle du cinéma, qui permet de reconstruire des situations en action par le biais du montage, comme si les regardeurs pouvaient y assister en direct ${ }^{28}$. L'effet est soutenu par la possibilité du public populaire de se reconnaitre dans les foules qui animent le film, car il s'agit de figurants qui ont été recrutés parmi le peuple ordinaire d'Odessa, où se déroule l'épisode du Cuirassé qui participa de la révolution russe de 1905. Le théoricien allemand esquisse finement la possibilité de retrouver une situation présente et vivante dans le Cuirassé, à travers l'expérience collective qu'offre la séance de cinéma. Cela concrétise son idée d'une critique qui sauve, à travers la réappropriation de l'expérience sensible par un public qui n'est pas acritique, mais plutôt distrait. Cette lecture amorce ensuite l'essai parisien L'oeuvre d'art à l'époque de sa reproductibilité technique. L'approche benjaminienne veut sauver la critique et conceptualise une critique qui sauve, alors que les assertions de Traverso rabattent cet art sans autre procès vers une "tradition soviétique". Non seulement Benjamin discute Le Cuirassé après avoir constaté en 1927, à Moscou, qu'il faut sortir du cadre soviétique, mais à la même époque, Trotsky s'inspire de la méthode de montage du film d'Eisenstein pour écrire son Histoire de la révolution russe (1930) à l'encontre de l'historiographie soviétique et sa tradition stalinienne ${ }^{29}$. Plus récemment, Alexander Kluge, formé par Adorno à ses débuts, a fait un usage vivant de l'héritage d'Eisenstein, en fabriquant un film de sept heures à propos du Capital de Marx, librement inspiré des notes que le réalisateur russe avait préparées de son vivant sur ce thème ${ }^{30}$. La preuve que l'intention critique ne se subsume pas à la tradition soviétique est que ce projet de 
film eisensteinien fut alors retoqué par Staline, aussi bien que par les boites de production de Hollywood.

Si le rapport de Benjamin à la tradition soviétique n'est pas précisé, Traverso voudrait quand même l'inscrire dans une "tradition marxiste", qui n'est pas spécifiée non plus. Pour Benjamin, en tout cas, le marxisme traditionnel est à l'origine d'un conformisme, qui est déjà refusé par Marx lors de son commentaire du programme de Gotha du parti ouvrier allemand, en $1857^{31}$. La thèse XI sur le concept d'histoire souligne ce conformisme marxiste ${ }^{32}$, qui ne se souvient pas des irruptions que furent la révolte des esclaves à l'époque de Spartacus (thèse XII), ni de la révolution française (XIV), ni du Vormärz qui précède la révolution internationale de 1848 , ni de la révolution des conseils allemande, mais qui a favorisé la soumission intellectuelle à des appareils de parti incontrôlés. Rappelons que le reproche principal de Benjamin à l'égard des mélancoliques de gauche, qu'il déteste, est leur conformisme.

Focalisé sur la tradition et les traditions, Traverso tient à assimiler Benjamin à une autre tradition encore, celle que Hannah Arendt a appelé la "tradition cachée" du judaïsme, qui est très riche en effet, l'associant alors à Henri Heine, Bernard Lazare, Kafka, Chaplin. ${ }^{33}$ Notons que les deux traditions, marxiste et cachée, ne se recoupent pas tellement, car aucun des auteurs de la seconde série ne se disait marxiste, pas plus qu'Arendt. Et Walter Benjamin n'apparait nulle part dans le livre d'Arendt. Elle n'y défend à aucun moment l'internationalisme de Marx ou de Benjamin, mais discute le projet politique d'un Etat national porté par des Juifs. C'est en ce sens politique très précis qu'elle souhaite reconsidérer le sionisme $e^{34}$. Cette discussion, précise sur le plan politique et complexe au niveau philosophique, ne peut pas être développée de manière satisfaisante dans le cadre du présent article (elle est aussi absente du livre de Traverso). Autre écart, l'appréciation arendtienne des conceptions de Heidegger est opposée au projet de Benjamin qui, lui, veut désintégrer Heidegger. Dans le volet de sa réflexion concernant les Lumières et la "question juive", Arendt se sert de concepts heideggériens pour mettre en doute la portée du projet humaniste de Herder, tout à fait à l'opposé de Benjamin ${ }^{35}$. En revanche, la théoricienne politique méprise les intellectuels allemands qui ont collaboré avec les Nazis (les Kästner), et elle regrette en outre la posture nostalgique et apolitique de Stefan Zweig qui se montre incapable de haïr les Nazis. ${ }^{36}$ Sur ce point précis, elle s'approche donc de l'attitude de Benjamin, puisque tous deux se positionnent à l'encontre d'une mélancolie impuissante (ce que Traverso ne voit pas). Par contre, Benjamin n'a pas du tout la même conception que Arendt, ni en rapport à l'internationalisme, ni concernant Heidegger, ni même concernant les précieux auteurs qui apparaissent dans la "tradition cachée". Si Benjamin apprécie Chaplin, il le situe précisément dans une même série comique américaine, avec Mickey Mouse ${ }^{37}$. Il est tout à fait possible que Traverso n'ait jamais lu précisément les textes de Benjamin qui sont en jeu, au sujet de Charlie Chaplin, Mickey Mouse, de la mélancolie de gauche de Kästner, du Cuirassé Patiomkine de Eisenstein ou encore au sujet de la critique de l'ontologie heideggérienne. Il est également probable qu'il n'ait jamais aperçu les contradictions majeures, entre les approches arendtiennes et benjaminiennes, pour nous servir finalement un mauvais cocktail. 


\section{Désintégrer Heidegger, contre l'ontologie et la théorie traditionnelle}

18 Le problème théorique plus fondamental qui pointe derrière ces improvisations touche à la philosophie politique de Benjamin, qui est entièrement dédiée à la critique de la tradition et de l'historiographie dominante, à la critique radicale de toute ontologie traditionnelle et de l'ontologie de Heidegger en particulier, puis complétée par la critique de l'ontologie soviétique qui veut justifier le développement durable d'un Etat dans un seul pays. L'intellectuel révolutionnaire Benjamin, lui, refuse de s'enrôler dans quelque tradition que ce soit : à chaque époque, il nous faut essayer de reprendre en main la transmission, une nouvelle fois, face au conformisme qui est en passe de s'imposer à elle ${ }^{38}$. Dans ses thèses sur le concept d'histoire, Walter Benjamin parle d'un accord secret qui relie la génération présente, vivante, aux générations passées, et cette approche se passe en dehors de la tradition. Les voix à qui nous prêtons aujourd'hui l'oreille, ne portent-elles pas l'écho de ceux qui ne peuvent plus parler ${ }^{39}$ Accepter cet héritage comporte une responsabilité, une exigence, qu'il est difficile d'écarter d'un revers de la main et encore plus difficile à satisfaire. Ce chemin implique un travail critique, qui n'est pas donné par avance, une confrontation à l'héritage. L'avantage de ce travail est de d'approcher une possible libération, alors que la posture de l'autruche mène sûrement à une souffrance que l'attitude acritique espère justement éviter. L'attitude benjaminienne, qui n'accepte aucune tradition donnée par avance, mais qui regarde toutes les expériences historiques dans leur richesse, comporte une grande potentialité créatrice, à partir des ressources immenses qui nous sont léguées, même lorsque la possibilité de réalisation peut sembler faible par moment. Benjamin veut faire éclater la tradition, alors que Traverso souhaite valoriser des traditions. La Théorie critique s'affirme justement à l'encontre de toute théorie traditionnelle, comme le souligne l'article fondateur de Max Horkheimer Théorie traditionnelle et théorie critique. ${ }^{40}$ Alors que Benjamin élabore (aux côtés d'Adorno) une critique de l'ontologie de l'être - contre Heidegger -, Traverso prétend inscrire l'exilé parisien dans une ontologie de l'être. Le leitmotiv des thèses de Benjamin sur le concept d'histoire est l'idée qu'il faille faire éclater le continuum de l'histoire, expression qu'il répète plusieurs fois. Adorno, qui a souligné qu'il se reconnaissait mot pour mot dans ces arguments, reformule cette même idée après la guerre : La tradition se définit aujourd'hui comme une ardente obligation de trouver une forme nouvelle, une articulation poussée et cette obligation ne tolère rien qui soit donnée par avance de façon traditionnelle. Celui qui se soustrait à cela fuit le caractère inachevé de l'histoire qui continue à le solliciter. ${ }^{41}$

19 La proximité intellectuelle entre Benjamin et Adorno, leur affinité élective qui se nourrit d'une grande amitié pendant une vingtaine d'années, ne fait aujourd'hui plus aucun doute. J'ai pu m'en convaincre lors d'un séjour de recherche aux Archives AdornoBenjamin à Berlin en 2015, et la correspondance des deux amis en témoigne à elle seule $^{42}$. Un leitmotiv central, peu remarqué, de leur complicité apparait à Paris dès la victoire d'Hitler, à savoir l'idée qu'il faille contrer Heidegger à tout prix. Benjamin veut "désintégrer Heidegger" et son ontologie anhistorique, aux côtés de Brecht ${ }^{43}$, un projet qu'il discute à partir des impulsions d'Adorno, dans un volet du Livre des passages qu'il élabore à Paris en $1935^{44}$. Benjamin, après avoir cité des travaux philosophiques d'Adorno, y constate que Heidegger s'obstine, en vain, à rendre abstrait le mouvement historique (la lutte des classes), en cherchant à imposer un jargon terminologique autour de "l'historialité", dans Etre et temps, en lieu et place de l'histoire réelle. Cela même avant de jurer allégeance à Hitler. Benjamin va contrer cette imposition 
abstraite par l'idée vivante d'images dialectiques, capables de mettre en relation l'imaginaire utopique d'une époque et l'action collective concrète, émancipatrice. Son interprétation d'Eisenstein peut illustrer cette approche, riche et originale, alors que la rédaction de son essai sur l'oeuvre d'art à l'époque de sa reproductibilité coïncide avec les travaux sur Paris et ses passages. La Commune de Paris est l'une de ces expériences concrètes qui rencontrent un imaginaire utopique. Ainsi, l'image dialectique écarte et renverse la notion plate de la vision du monde, que Heidegger a pu exploiter parce qu'elle reste indéterminée chez Husserl (comme le remarque alors Benjamin).

Les thèses sur le concept d'histoire de Benjamin sont le point d'orgue de sa réflexion sur ces sujets, tournés en pratique contre le fascisme, l'autoritarisme de Carl Schmitt et la mystification anhistorique de Heidegger. L'éditeur scientifique de Benjamin, Tiedemann, a fait l'effort de restituer la globalité et la cohérence de l'élaboration benjaminienne et sa complémentarité avec la pensée d'Adorno, puis sa prolongation dans la critique de Heidegger qui constitue la Dialectique négative, à travers un exposé éditorial de 120 pages $^{45}$. Les essais et écrits parisiens s'avèrent toutes issus du Livre des passages, qui se condense finalement dans les thèses sur le concept d'histoire. Le jour de sa mort, Benjamin confie une brève lettre à ses compagnons, adressée à Adorno, où il exprime sa dernière volonté de lui transmettre ses pensées, qui sont contenues dans les désormais célèbres thèses sur le concept $d$ 'histoire. Adorno découvre plus tard ce manuscrit, et constate, dans un hommage à Benjamin, qu'il venait auparavant d'utiliser les mêmes mots dans un autre texte, saisissant presque mot pour mot le sens conceptuel du testament intellectuel de Benjamin. ${ }^{46}$ Dans les années 1960, Adorno parvient enfin à reprendre ce lourd chantier, en précisant la critique de Heidegger, de l'ontologie fondamentale et de l'historialité abstraite, qui correspond à une pensée foncièrement autoritaire. ${ }^{47}$ Ainsi, il parvient finalement à désintégrer Heidegger, dans le droit fil de l'esprit benjaminien qui est souligné dans Dialectique négative ${ }^{48}$. L'éditeur allemand de Heidegger, Peter Trawny, a finit par constater que Adorno a tué Heidegger ${ }^{49}$. Autrement dit, Adorno a réalisé le projet benjaminien consistant à désintégrer Heidegger. A l'endroit exact du Livre des passages, où Benjamin s'appuie, déjà, sur la philosophie adornienne pour défaire l'abstraction historique mortifère de Heidegger, émerge l'alternative à la fausse représentation d'un être originaire et inaccessible, celle que Benjamin nomme l'image dialectique. L'expérience vivante et l'imaginaire utopique peuvent entrer en mouvement, à travers les luttes des classes, à l'instar d'une image qui associe les deux dimensions, pratique et intellectuelle. Le meilleur indice que Traverso ne saisit pas cette pensée dialectique, lorsqu'il évoque brièvement le concept benjaminien d'image de pensée (Denkbild), est qu'il ne cite pas la discussion AdornoBenjamin au sein du Livre des Passages, où l'image dialectique précise et complète l'image de pensée. L'image dialectique promet ici de désintégrer Heidegger, l'ontologie traditionnelle, l'abstraction historiale de l'être, et le nazisme qui porte ces discours en pratique.

21 Même si Traverso n'a en rien saisi ce développement historique et intellectuel, décisif, qu'il ne mentionne nulle part, il est regrettable que sa présentation française à la correspondance Adorno-Benjamin ait été mise au pilon, en 2003 (La Fabrique), pour ne pas avoir respecté les recommandations du Archiv Adorno-Benjamin associé à la fondation Adorno. Opposé à toute forme d'occultation et toute forme de pilonnage, j'ai consulté ce texte écarté, qui préfigure au fait toutes les méprises du livre Mélancolie de gauche, texte qui se trouve d'ailleurs reproduit dans une version allemande du même livre. Une recension de cette version a bien résumé le cas Traverso : Sa critique 
bienpensante ne devient pas plus véridique par le simple fait de répéter de vielles lunes dont la fausseté a été démontrée, mais qui se maintiennent malgré tout. (...) De manière globale, nous conseillons à Traverso de lire plus sérieusement la correspondance entre Adorno et Benjamin..$^{50}$

En effet, Traverso tente de construire une division artificielle, entre Benjamin et Adorno, sans rien démontrer. L'auteur s'emploie à dénoncer une sorte de paternalisme dominateur d'Adorno sur Benjamin dans les années 1930, arguant que le premier aurait imposé ses arguments grâce à la dépendance matérielle du second. Heureusement, le contenu de leur correspondance prouve exactement le contraire à qui veut bien la lire. En 1935, Adorno assure Benjamin qu'il va défendre en bloc le financement de son projet d'un Livre des passages (Paris, Capitale du 19ème siècle), avant même d'ouvrir un dialogue philosophique plus approfondi: Je vais écrire au plus vite à Horkheimer et demander l'acceptation du travail en bloc, et du même coup aussi, bien sûr, son financement. ${ }^{51} \mathrm{I}$ ajoute ici que le projet parisien illustre l'étroite communication entre nos pensées, par delà l'Atlantique qui les sépare. Comme le montre une lettre d'Adorno à Horkheimer, datée du 8 juin 1935, il tint aussitôt parole et Benjamin obtint un financement qui lui assura une vie indépendante des structures et revues européennes. La thèse d'une volonté dominatrice ou d'un chantage intellectuel d'Adorno est infondée, en ce sens malveillante. Dès 1939, Gretel et Theodor Adorno pressent Benjamin de quitter Paris, soutenant sa fuite quand leur ami Walter est enfin prêt à accepter leur aide en 1940.

Après avoir tenté de diviser Adorno et Benjamin, pour mieux régner, Traverso voudrait encore qualifier Adorno de romantique conservateur, dans sa malheureuse tentative de présentation de leur correspondance. Le romantisme conservateur est un qualificatif infondé, au vu des préférences littéraires et philosophiques du théoricien critique, mais relève d'un vocabulaire généralement associé aux sources du fascisme dans le débat académique. De manière confuse, ce vocable suggère encore cette prétendue proximité entre la Théorie critique et les Nazis que le stalinisme a fabriqué de toute pièce du vivant de Staline.

Cette fausse piste nous éloigne de la compréhension dialectique, qui habite Benjamin, et de sa critique de l'ontologie traditionnelle (romantique-réactionnaire) ou heideggérienne-nazie. Lors des festivités parisiennes pour les 150 ans de la révolution française, Benjamin produit par exemple la traduction d'une lettre de Herder, qui dénonce le nationalisme romantique allemand, celui qui se nourrit d'une fantasmagorie du lien du sang ou de la race. En relançant la pensée cosmopolite vivante de Herder, Benjamin entend faire jaillir l'expérience de la révolution française et de ses répercussions internationales, alors que les mouvements fascistes et racistes se font menaçants. ${ }^{52}$ L'exemple concrétise l'approche que Benjamin esquisse dans ses thèses sur le concept d'histoire: transmettre la critique en rendant vivante les luttes du passé, malgré l'histoire officielle, écrite par les vainqueurs.

Contrairement à Benjamin et Adorno, qui comprennent la gauche de manière dialectique, comme un principe critique opposé à la tradition et la vision ontologique, Traverso se propose justement de définir la culture de gauche en tant que tradition, d'un point de vue ontologique. Cela lui permet d'associer un auteur inspiré de Heidegger comme Giorgio Agamben à sa démarche, dont l'éloignement de la Théorie critique est évident..$^{53}$ Traverso, qui rend hommage à Daniel Bensaïd dans sa Mélancolie de gauche n'a t'il pas saisi pourquoi le second nomme Benjamin L'Anti-Heidegger de l'entre-deux-guerres? ${ }^{54}$ Tout le projet francfortois se construit à travers une critique de l'abstraction ontologique de Heidegger, depuis les écrits parisiens de Benjamin jusqu'à 
la Dialectique négative d'Adorno. La pensée de Benjamin est une critique de la tradition et n'a rien à voir avec une "mémoire marxiste" univoque, raison pour laquelle il résiste de son vivant à toute "tradition marxiste" 55 . Traverso veut en faire un chainon de la tradition, alors que les Thèses sur le concept $d$ 'histoire ne cessent de clamer que la critique implique de sortir de la tradition. Benjamin pense et écrit que la véritable image historique est fugace: Das wahre Bild der Vergangenheit huscht vorbei (thèse $\mathrm{V}$ sur le concept d'histoire), l'image historique est image dialectique. En ce sens, il demeure obscur dans le livre Mélancolie de gauche en quoi l'approche originale de Benjamin s'opposerait à la dialectique négative, comme l'affirme Traverso, et pourquoi une telle dialectique non-traditionnelle serait "mutilée"?56 La vie mutilée fait apparemment référence au sous-titre français des Minima moralia (Réflexions sur la vie mutilée), où Adorno développe justement une position critique, non-stalinienne, qui correspond à l'élan fondateur de l'Institut de Francfort, pour ne pas être déformé et mutilé intellectuellement, malgré les dommages et traumatismes subis pendant la guerre. Le sous-titre allemand évoque plutôt une vie endommagée ${ }^{57}$. La même position, claire, s'exprime dans les études pour un nouveau Manifeste communiste que mènent Horkheimer et Adorno en 1956, au moment de la chute du modèle stalinien ${ }^{58}$.

Ce qui a "mutilé" ou endommagé la pensée dialectique d'Adorno et Benjamin fut le discours ontologique du marxisme soviétique, et la propagande stalinienne qui voulait présenter ces auteurs comme étant proche des Nazis, comme étant des contrerévolutionnaires apolitiques et des défaitistes. Dans un livre lumineux et salutaire, $L e$ marxisme soviétique, Herbert Marcuse à parfaitement saisi comment la mentalité stalinienne a détruit toute pensée critique et dialectique, vivante, en prétendant que la lutte des classes serait close en Union soviétique, interdisant de penser les conflits entre la paysannerie, la classe ouvrière urbaine, l'intelligentsia et la bureaucratie $\mathrm{d}^{\prime} E \mathrm{Etat}^{59}$. Sur le plan théorique, ce refoulé a produit l'assertion qui voulait qu'il n'y aurait plus de contradiction conflictuelle sous l'Etat soviétique, mais exclusivement des contradictions non-antagoniques. Presque logiquement, la dialectique des conflits se trouve anéantie, et un discours du développement continu de l'Etat prend sa place, une ontologie soviétique qui est bordée par l'année de création de l'URSS et son but ultime, la pérennisation du stalinisme (en réalité sa dissolution interne en 1956 et la dissolution de l'Etat soviétique en 1991). Le tournant prosoviétique obligé de Lukacs, qui débute à Moscou en 1933, n'implique non seulement son virage pamphlétaire à l'encontre de la Théorie critique, mais aussi son abandon de la pensée dialectique au profit d'un discours ontologique. Les trotskystes se sont brisés sur la question de savoir s'il fallait défendre l'Etat soviétique de manière inconditionnelle (comme le préconisa Trotsky), ou bien de le soumettre à une critique radicale, dialectique, à l'instar de Cornelius Castoriadis, Miguel Abensour ou encore Jean-Marie Vincent. Souvenons nous que Boris Fraenkel, le mentor de Jospin ou Mélenchon, fut exclu de son parti trostkyste-lambertiste après 1968, pour avoir osé traduire et publier le premier texte de Wilhelm Reich en France.

\section{Dissidence ou répétition?}

La question reste de savoir comment un historien comme Traverso revisite sa propre tradition politique, dans la mesure où sa fiche wikipédia anglophone le présente comme historien communiste (consulté en mai 2020), tandis que la fiche germanophone précise son appartenance ancienne à la Ligue communiste révolutionnaire, pendant qu'il 
affiche sa proximité avec l'intellectuel trotskyste Daniel Bensaïd, auquel il dédie un chapitre de sa Mélancolie de gauche. Ce questionnement de l'héritage peut être affiné à travers la discussion d'un programme trotskyste de 1992, dont le titre fait déjà discrètement référence au bref texte de Benjamin Mélancolie de gauche. Le manifeste de la LCR de cette année-là, qui signe l'effondrement de l'Union soviétique, est intitulé : A la gauche du possible. Comme le passage de Benjamin que nous avons cité plus haut le signale, l'expression à la gauche du possible se niche dans son propre texte contre la mélancolie de gauche. Le mot moqueur à la gauche du possible (qui ne définit aucune prose de position politique concise selon Benjamin) fut sans doute introduit en titre du manifeste trotskyste par Daniel Bensaïd, qui venait alors de découvrir les écrits et idées de Benjamin. Le rapprochement n'est en rien fortuit, puisque Traverso rend longuement hommage à Bensaïd dans sa Mélancolie de gauche ${ }^{60}$. Connaissant la fibre léniniste de Bensaïd, qui fit partie des rédacteurs des textes programmatiques de la LCR, l'allusion "à la gauche du possible" comporte sans doute une dose ironique ${ }^{61}$. Car le manifeste trotskyste de 1992 s'exclame : Les idéologues nécrophages célèbrent la mort du marxisme, la mort du féminisme, la mort du communisme, voir la mort du socialisme. Pourquoi avoir attendu qu'à Moscou les statues aient étés renversées et le drapeau rouge abandonné pour proclamer la mort du communisme? Si le communisme devait mourir des crimes perpétrés en son nom, cela ne daterait pas d'aujourd'hui, ni d'hier ${ }^{62}$.

Là où Bensaïd s'insurge contre l'air du temps morbide, cet esprit du Thanatos, qui célébra la mort du féminisme, du communisme ou du socialisme, autant que le prétendu naufrage de la révolution française, le constat mélancolique de Traverso laisse faire. Pour concrétiser ce que signifie une mélancolie de gauche, l'auteur proclame sans ambages "la fin du communisme", et "la fin du féminisme socialiste", donc leur mort $^{63}$. Cela tout en valorisant au passage le texte de Carla Lonzi Crachons sur Hegel qui célèbre, elle, l'aristocrate Olympe de Gouges, guillotinée pour avoir encouragé l'insurrection contre la république révolutionnaire, après avoir valorisé Marie Antoinette (elle en partagea le sort tout comme son avocat, Tronson Ducoudray). Benjamin, lui, avait discuté la féministe marxiste Alexandra Kollontaï, qui ne s'inscrit certainement pas dans la tradition contre-révolutionnaire d'Olympe de Gouges. Aujourd'hui, le féminisme socialiste et l'esprit du Manifeste communiste de 1848 sont portés par des autrices comme Cinzia Arruzza, Tithi Bhattacharia et Nancy Fraser, pour ne citer que ces trois philosophes, qui sont vivantes ${ }^{64}$.

La mélancolie de Traverso le pousse à enterrer le communisme, qui serait fini, malgré les ressources du Vormärz d'avant 1848 et du Manifeste communiste qui irriguent la pensée de Rosa Luxemburg, de Benjamin, de Brecht ou de Bloch. Là où il essaie de rester fidèle à l'intellectuel trotskyste Bensaïd, Traverso répète ses erreurs, en particulier lorsque Benjamin se trouve enrôlé dans une tradition marxiste - dont le droit fil irait de Marx à Trotsky. Pourtant, Marx n'était pas marxiste ${ }^{65}$. Et Benjamin n'était pas trotskyste, comme Michael Löwy l'avait déjà amicalement rappelé à Bensaïd $^{66}$. L'erreur de Traverso se nourrit probablement du livre bensaïdien Le pari mélancolique, où apparait la question de savoir pourquoi "tous les intellectuels révolutionnaires" seraient mélancoliques, dépressifs, sinon suicidaires ${ }^{67}$ ? L'un des exemples qui se trouve dans ce livre est Tucholsky, suicidé, que Benjamin range effectivement parmi les mélancoliques de gauche, mais qu'il s'agirait de ne pas suivre. Sans examiner la mélancolie de Bensaïd, ni le cas très limite de Benjamin (qui cherche d'abord à fuir pour survivre et non pas à se suicider), la règle qui voudrait que tous les 
intellectuels révolutionnaires soient dépressifs ne résiste pas à l'examen. Parmi les 21 membres du séminaire de l'Institut de Francfort de mai 1923, aucun n'a fini ou mené sa vie de manière foncièrement dépressive. Karl Korsch par exemple lutta jusqu'à son dernier souffle aux USA. Richard Sorge fut exécuté par les fascistes au Japon, après avoir réussi à dévoiler leur stratégie militaire, ce qui permit par ricochet la défense efficace de Moscou en 1941 (par l'envoi des troupes soviétiques sibériennes). De son côté, Lukacs fut obsédé par sa survie, et non pas par le suicide. Les intellectuels critiques vivants, liés à l'héritage francfortois, dont Angela Davis, John Holloway, Nancy Fraser ou encore Oskar Negt ne montrent aucune tendance suicidaire. A 85 ans, Negt a publié une autobiographie sur le thème de la chance de vivre ${ }^{68}$. Idem pour les membres du comité éditorial de la revue Socialisme ou barbarie, qui est loin de se présenter comme un vivier de suicidaires, ou encore le mouvement surréaliste qui est tourné vers la créativité et la vie. Il n'est pas vrai que tous les intellectuels radicaux veulent se noyer dans la piscine publique, à la manière d'un Kästner.

Nous l'avons vu, Traverso ne saisit pas de manière précise les héritages qu'il invoque, mais dont il prétend néanmoins faire une tradition. Il ne comprend pas clairement la brisure de la tradition soviétique, ni la tentative critique qui émane du manifeste coécrit par Bensaïd au moment de la chute de l'Union soviétique. Dans ce cadre, Traverso accumule les contre-sens à propos de la critique benjaminienne de la mélancolie de gauche, de la méthode de montage d'Eisenstein, ainsi que de l'usage qu'en fait le Trotsky exilé. Le passage au postmarxisme qu'esquisse Traverso, au détour de son hommage post mortem à Bensaïd, se passe de toute introspection critique, dans une attitude qui évacue les problèmes irrésolus du passé (pointés par les thèses benjaminiennes sur le concept d'histoire). Cela tout en maintenant une position de surplomb intellectuelle qui puise son capital culturel dans la "tradition marxiste" et l'hommage aux intellectuels fondateurs, morts. Le postmarxisme est un discours post mortem. Le "post" du postmarxisme ou du poststructuralisme fonctionne alors comme une ellipse, une coupure avant-après, qui fut pourtant catégoriquement retoquée par Foucault, Derrida ou Bensaïd de leur vivant. En ce qui concerne Bensäid, il n'a jamais gouté le postmarxisme, ni le discours althussérien qui en est l'un des fondements. Il est donc assez douteux de faire de Bensaïd un passeur du postmarxisme à la française, de Foucault ou de Deleuze en particulier, dans la mesure où l'intellectuel trotskyste n'a cessé d'affirmer ses désaccords philosophiques et politiques avec ces auteurs de son vivant $^{69}$. Pour sa part, Foucault ne s'est jamais dit marxiste de son vivant, tout en se moquant ouvertement d'une spéculation poststructuraliste, qui ne veut rien dire d'après lui ${ }^{70}$. A côté de l'opposition à tout stalinisme intellectualisé, la ligne intellectuelle de Bensaïd fut fondée sur le refus catégorique de l'optique ontologique qui se retrouve chez Althusser ou Foucault (tous deux impressionnés par Heidegger après la guerre), rappelant ainsi la véritable position benjaminienne: Heidegger ontologise et resacralise. Marx désacralise et désontologise ${ }^{71}$.

31 Manifestement, la lecture directe des textes et auteurs ne permet pas toujours de comprendre l'usage qu'en fait Traverso. Le regard sociologique de Benjamin pourrait offrir une piste explicative à ce type de prisme trouble. L'exilé parisien saisit, en effet, l'arrivisme des groupes d'écrivains qui se tiennent à l'écart des luttes sociales, pour transformer les figures prolétariennes et cultures oppositionnelles en pastiches vendeurs. Benjamin remarqua que les mélancoliques de gauche sont des parvenus d'une petite bourgeoisie nouvelle, poussés par le "désir d'un mécène" disposant de très grandes sommes d'argent, avant de préciser le rôle social de cette tendance : La fonction 
de cette intelligentsia est d'engendrer, sur le plan politique, des coteries au lieu de partis, des modes à la place d'Ecoles du point de vue littéraire, et des agences à la place de producteurs, d'un point de vue économique. (...) Le fatalisme de ceux qui sont placés le plus loin du processus de production, et dont le sombre démarchage indexé sur la conjoncture est comparable à l'attitude d'un homme qui s'abandonne sans rétention aux soubresauts fortuits de sa digestion. Sans nul doute, le grondement de ces vers tient plus du ballonnement que de la subversion. Depuis toujours, le cynisme et la mélancolie font bon ménage, mais depuis que les fluides corporels se sont mis à stagner, l'abattement spirituel s'étale dans toutes les rues. ${ }^{72}$

Pierre Bourdieu avait cherché à exposer l'attitude de ce groupe social, dans un chapitre consacré à la nouvelle petite bourgeoisie qui touche aux métiers de l'édition, du design et de la communication, aux nouvelles niches universitaires. ${ }^{73}$ Cet effort reste à actualiser. Ici, il ne s'agit pas de dresser une analogie trop rapide, entre les mélancoliques de gauche berlinois des années 1930 et ceux qui s'y identifient presque un siècle plus tard. Pour avancer dans la compréhension des motifs et motivations contemporaines, il s'agirait de partir du constat que la galaxie poststructuraliste, dont les professeurs référents gagnent en moyenne 200.000 Dollars par an sur les campus US de la côté Est (Ivy League), est bien implantée dans les spécialisations universitaires tout comme le champ éditorial, alimentée par le mécénat privé et les fondations ${ }^{74}$. Benjamin n'est plus là pour répliquer à ses détracteurs contemporains, et personne ne peut parler à sa place, alors que sa critique mériterait une actualisation matérialiste, lorsqu'il remarque que la position mélancolique... perd rapidement en force de frappe face à la grande bourgeoisie, avant d'avouer son désir du mécène en un râle : "Ô, si seulement nous avions une douzaine de sages, pourvus de très grandes sommes d'argent". Cela n'étonnera personne que Kästner affiche une familiarité tordue qui va de pair avec une manière de voir l'économie de travers, lorsqu'il il veut régler son compte aux banquiers dans un "Hymne". ${ }^{75}$

Walter Benjamin pensait que la lutte des classes, que Marx avait mieux saisi que les marxistes français, est une lutte autour de choses brutes et matérielles, lutte sans laquelle les éléments les plus fins et raffinés ne peuvent pas émerger (thèse IV sur le concept d'histoire). La contre-culture des opprimés maintient cet héritage sous la forme de différentes qualités non-académiques que sont l'humour, le courage, la ruse, la persévérance critique (thèse IV), et qui ne résident pas dans le marxisme soviétique ou traditionnel. Le genre de postmarxisme mélancolique dont se sert Traverso, parmi d'autres discours, est une manière comme une autre de contourner le travail de deuil historique, une manière de ne pas éprouver la perte des modèles et références du passé. Dans la galaxie post-structuraliste, la grande époque des écrits staliniens de Louis Althusser (qui se réfère encore à Staline en personne dans Pour Marx) sert toujours de modèle théorique général, repris sous la forme de conceptions anhistoriques, sans en rien mettre en question les fondements théoriques mêmes qui se trouvent chez Althusser. ${ }^{76}$ L'idée althussérienne de surdétermination (lancée dans Pour Marx en 1965) s'applique par exemple, chez Balibar, au racisme, à l'antisémitisme et au nationalisme, sans aucun retour critique sur la conception originaire de cette fameuse surdétermination. ${ }^{77}$ Balibar annonce dans le même texte qu'il faut se délester du verbiage marxiste-léniniste de la grande époque althussérienne, mais il semble ignorer qu'Althusser tire ses arguments et notions de Staline, Mao Tsé Toung ou encore d'une réception trop approximative du docteur Freud. L'argument principal de l'original althussérien dans Pour Marx est appuyé sur une citation de Mao de 1927 qui postule alors que le socialisme ne comporte aucun conflit mais se base sur des contradictions non-antagoniques. En l'absence d'un retour critique, le passage du marxisme dogmatique 
au postmarxisme (ou au poststructuralisme) demeure obscur. Stuart Hall part, lui aussi, de ce même texte althussérien, portant sur la contradiction et la surdétermination (Pour Marx), pour lancer sa théorisation postcoloniale ${ }^{78}$. Le caractère anhistorique de cette construction saute aux yeux, dans la mesure où Althusser, né en Algérie, n'a jamais commenté ou soutenu la révolution algérienne de 1954-62, et fut totalement absent de la grève générale de Mai 68. Pourtant, les éléments raffinés et spirituels ne peuvent se comprendre en dehors de la lutte des classes la plus brute, selon Benjamin. La lutte des classes, observable de manière empirique et historique, disparait chez Althusser au profit de spéculations théoriques, tout comme chez Traverso. L'exemple le plus net de son incompréhension se manifeste dans un pur jeu d'esprit, au sujet du postcolonialisme, où l'auteur mélancolique postule que la Théorie critique d'inspiration francfortoise n'aurait jamais rencontré le marxisme noir (black marxism). ${ }^{79}$ C'est absolument faux, puisque Angela Davis a été entièrement formée par Herbert Marcuse jusqu'à son doctorat à l'Université de Californie, formation rythmée par un séjour à Francfort où elle a suivi les cours de Theodor W. Adorno et Oskar Negt. Son livre Femmes, classe, race témoigne lumineusement de cette formation et de cette rencontre fructueuse, qu'elle revendique ${ }^{80}$. Dans la mesure où Davis aborde les relations conceptuelles de manière historique, à partir de la lutte des classes aux Etats Unis (de 1848 à 1978), son approche échappe au schéma anhistorique des études poststructuralistes et postcoloniales. C'est probablement la raison pour laquelle Traverso l'ignore complètement, mais s'autorise à spéculer sur un prétendu eurocentrisme de la Théorie critique internationale. Ajoutons que John Holloway, Fernando Matamoros et le commandant Marcos ont utilisé la Théorie critique au milieu de la jungle lacandon, au sein d'un mouvement zapatiste qui se veut intergalactique ${ }^{81}$. Le chercheur et docteur kurde Muharren Acikgöz a, lui, souligné la permanence de la Théorie critique ${ }^{82}$. Chez Traverso, le postcolonialisme théorique remplace les luttes anticoloniales réelles, tout comme les spéculations sur Benjamin remplacent ses écrits réels.

Pour sa part, Jacques Derrida avait souligné dans Spectres de Marx que la condition d'un renouveau critique et internationaliste était de faire le deuil du marxisme traditionnel, de facture soviétique, et en particulier de ce marxisme français "autour d'Althusser". Sa critique rejoint sans le dire celles de Jacques Rancière, d'E.P. Thompson, de Cornelius Castoriadis, de Jean-Marie Vincent et de Daniel Bensaïd ${ }^{83}$.

Derrida, que Traverso voit comme une ressource du postmarxisme, se situe en réalité à l'opposé exact des théorisations postmarxistes ou postcolonialistes qui répètent les schémas de base althussériens. Derrida partage ce constat au moment même où il écrit un livre pour Benjamin - contre Carl Schmitt - et où il met péniblement en doute la tradition heideggérienne ${ }^{84}$. Ce travail de deuil, douloureux, difficile, inachevé, qui passe aussi par une réappropriation hésitante de Freud, permet à Derrida de dire : Je voudrais commencer à vivre enfin ${ }^{85}$. Au même moment, Judith Butler reste bloquée dans la discussion de la mélancolie freudienne (de 1915), sans jamais passer à la libération, ni aux écrits révolutionnaires de Freud autour de la psychologie de masse, motivés par sa joie de voir arriver la révolution des conseils. ${ }^{86}$ Contre l'avertissement explicite de Foucault ${ }^{87}$, Butler s'efforce de définir le passage théorique du structuralisme au poststructuralisme, à travers des phrases très longues et inaccessibles qui lui ont valu le grand prix de la pire écriture philosophique aux USA en $1998^{88}$. La philosophe a aussi développé des thèses sur le pouvoir du discours, autour du terme althussérien d'interpellation, en omettant de rappeler que chez Althusser l'idée d'interpellation dépend littéralement de la "règle d'or de la pratique de la lutte des classes marxiste-léniniste". 
${ }^{89}$ Admirons la belle concordance du fond et de la forme, lorsque Butler reprend aujourd'hui l'inspiration althussérienne de Lire Marx, en abordant quelques textes de Marx dans les locaux de l'Ecole normale supérieure, jadis habitée par Althusser, réflexions publiées aux Editions sociales, historiquement issues du PCF. ${ }^{90}$ Si Althusser a avoué n'avoir lu que quelques textes du jeune Marx, au moment de Lire Marx (1965), Butler recommence tardivement la lecture du jeune Marx, dans une répétition presque parfaite ${ }^{91}$.

L'ellipse qui saute du marxisme au post-marxisme masque ainsi une certaine incapacité critique, qui s'accompagne de l'absence d'un travail de deuil et du refus de la potentialité psychanalytique. Chez Traverso, par exemple, cet aspect devient tout à fait explicite quand la mélancolie est retenue, contre l'impulsion benjaminienne, écartant toute dimension freudienne, tout en revendiquant le maintien du poids du passé, dont la perte définitive n'est pas envisagée : C'est la mélancolie de gauche qui ne veut pas se délester du fardeau, bien qu'il soit souvent lourd à porter ${ }^{92}$. Si Benjamin sentait qu'il fallait se débarrasser de la posture impuissante de la mélancolie de gauche, pour ouvrir de nouvelles perspectives, qui incluent la pensée freudienne, Traverso affirme littéralement qu'il veut maintenir la mélancolie et le poids du passé, pourtant si lourd à porter, sans trop cacher la punition de soi qui est inhérente à cette mélancolie. C'est peut être pour cette raison que Traverso ne cherche pas à sortir des traditions, qu'il lui est si difficile de concevoir le point de vue benjaminien d'une transmission vivante, non-ontologique, inspirée de l'idée freudienne du souvenir. C'est également une explication possible du fait que Traverso, qui s'est fait connaitre en tant qu'historien des idées, se situe à l'évidence loin des textes, sources et archives, concernant Benjamin et Adorno (qui tombent d'accord sur l'importance de Freud en 1935).

Aujourd'hui, certaines ramifications althussériennes, devenues postmarxistes depuis la mort d'Althusser et la chute du marxisme soviétique, inspirent un espace éditorial qui poursuit sa dynamique indépendamment de tout parti. Benjamin avait bien saisi ce caractère apolitique et volontairement impuissant. Le poststructuralisme fonctionne comme une compensation, un Ersatz des idéologies identitaires du 20ème siècle pour toutes les personnes qui préfèrent la répétition à la dissonance. La répétition de stéréotypes soviétiques à l'encontre de la Théorie critique répond à un besoin de se rassurer, un besoin ontologique et identitaire que Adorno avait exposé et soumis à la critique dans sa Dialectique négative. D'un point de vue de la Théorie critique, les éditions en poche d'essais postmarxistes, généralement inexacts mais prisés par un public para-universitaire, participent de la production stéréotypée d'une industrie culturelle. En ce sens, il s'agit d'une fausse représentation qui est illusoire (infondée scientifiquement et historiquement), mais réelle (en tant que livre-marchandise, croyance partagée et rapport social fétichiste). Le Ersatz de la critique réelle répond à une mentalité qui exprime un malaise dans la société bourgeoise, sans chercher à la dépasser, incapable de libérer la moindre puissance révolutionnaire. Benjamin précise que l'attitude des mélancoliques de gauche consiste à imposer à la dialectique révolutionnaire le visage d'un bon sens dépourvu de toute condition de classe ${ }^{93}$.

\section{Pour une gauche érotique}

Si Benjamin a si vivement pris parti contre toute mélancolie de gauche, et pour la perspective freudienne, la question reste de savoir quel serait le principe créatif qui puisse poursuivre ou amplifier cet élan critique. Ce n'est certainement pas une gauche 
benoitement optimiste ou euphorique, dans la mesure où la Théorie critique restait circonspecte face à tout optimisme naif - dans un genre de pessimisme gramscien qui n'a rien à voir avec l'accusation grossière de défaitisme dont elle a fait l'objet. Chez Freud, l'opposé dialectique à la mélancolie ou la dépression, happée par Thanatos, se trouve dans le principe créateur d'Eros. Une gauche érotique pourrait surgir de l'abandon de la gauche mélancolique, nihiliste et pseudo-radicale. Cette idée mérite développement, elle s'applique assez bien au sujet du repoussoir absolu que fut Kästner aux yeux de Benjamin. Kästner ne passe jamais à l'acte, refuse d'envisager l'expérience du bonheur ou de la jouissance, et ne propose que des substituts, des Ersatz. Le même Kästner bascule rapidement vers le Thanatos, en acceptant de divertir les nazis à partir de 1934, puis en travaillant dans l'industrie du spectacle dans l'après-guerre. Loin de cet opportunisme, qui avait auparavant poussé une série de mélancoliques de gauche à soutenir les officines de Staline, Benjamin s'est convaincu du caractère mortifère du stalinisme, à travers une rencontre amoureuse qui l'amena à Moscou en 1927. Ici, Alexandra Kollontai, qu'il cita parfois, avait inventé "l'amitié érotique" pour favoriser la sortie de la tradition, conventionnelle et répressive $e^{94}$.

L'opposition à une gauche mélancolique - et le passage à une gauche davantage érotique - s'applique assez bien au tournant révolutionnaire que négocia Freud en 1917. Comme je l'ai indiqué, il se trouve alors bloqué, après avoir écrit son essai sur la mélancolie, face aux horreurs de la guerre et la peur de voir mourir ses fils. C'est lors de la découverte des révolutions russe et allemande-autrichienne qu'il revit, qu'il se libère intellectuellement, en amorçant sa théorie de la psychologie des masses, qui ouvre de nouvelles perspectives. Ce moment créatif l'approche sans aucun doute d'Eros. L'opposition entre Thanatos et Eros reste en réalité encore trop schématique, car Freud esquisse des relations complexes qui se jouent entre trois pôles, au cours du développement de chaque personnalité. Le pôle obsessionnel (tourné vers le travail, la persévérance, l'abnégation), le pôle narcissique (tourné vers l'amour de soi sans lequel la survie n'est pas pensable), le pôle érotique (la disponibilité de recevoir l'amour d'un/ e autre ou des autres). Le nouveau modèle freudien, plus élaboré, est publié en 1931, exactement la même année que la critique benjaminienne de la mélancolie de gauche. ${ }^{95}$ Dans son hommage à Benjamin, Adorno saisit la pensée créative se son ami comme un don, un partage de son abondance, qui cherche à compenser ce que le conformisme et la discipline tendent à refuser : la jouissance. Du point de vue intellectuel de Freud, pareille ouverture qui reçoit le don amical comme une promesse de jouissance, s'approche du pôle érotique.

Transposé à la dynamique de la gauche, le pôle obsessionnel semble surinvesti depuis longtemps, par l'identification au travail productif, à la discipline collective, à la mobilisation presque militaire qui caractérise les partis, et par l'abnégation militante qui frôle parfois le martyre ou l'auto-flagellation, ainsi que par cette forme de rétention qui se fonde sur un deuil impossible. Le pôle narcissique existe bel et bien - la gauche se montre souvent fière de ses accomplissements, de sa moralité admirable, de sa beauté artistique ou intellectuelle, pouvant aller jusqu'à la fausse générosité qui renferme une attitude narcissique. Les intellectuels et intellectuelles de gauche, qui sont sans exception des êtres plus ou moins traumatisés, et qui veulent vivre ou survivre à travers une expression critique, sont nécessairement persévérants et obsessionnels par leur érudition et leurs écritures, mais moins sûrement narcissiques. Une partie de ces intellectuels peine à s'aimer; les écrits autobiographiques de Gorz, Sartre ou Althusser 
l'illustrent bien. ${ }^{96}$ Althusser est allé jusqu'à l'autodestruction, au terme d'une spirale maniaco-dépressive qu'il expose lui-même jusqu'au moindre détail. Il faut dire que chez Freud, les différents pôles sont pensés en rapport à des personnalités relativement stables, et non pas à partir d'intellectuels particulièrement traumatisés et fragiles. C'est à mi-chemin, entre obsession et amour de soi, que les caractères se stabilisent davantage, remarque-t-il, favorisant des personnalités capable de grandes réalisations culturelles.

41 Enfin, le pôle érotique de la gauche est manifestement sous-développé. L'amour en liberté de Fourier ou l'appel à la liberté sexuelle des femmes de Bakounine ont fait sourire, le renvoi de Marx au romantisme révolutionnaire de George Sand et de Henri Heine reste méconnu, le droit à la paresse de Lafargue fut suspect, la gauche érotique de Kollontai, Marcuse, Guérin ou Despentes ${ }^{97}$ reste dominée par les apparences plus austères de Lénine, Mitterrand ou Piketty. Make love not war fut un mot important, mais Le pouvoir est au bout du fusil reste marquant. La quête du pouvoir prime souvent sur l'enquête de l'amour. Le pôle érotique n'est pas purement libidinal ou sexuel - sachant que l'aspect directement sexuel est aussi présent dans les autres pôles, par exemple dans le plaisir masochiste (obsessionnel) ou masturbatoire (narcissique). La capacité érotique comporte une part de sublimation. La quête absolue de l'orgasme chez Reich a pu cacher la variété des plaisirs, et la consommation des actes de Catherine Millet ne dit rien de la jouissance. Le manifeste féministe de Arruza, Bhttacharia et Fraser met en garde contre le marché et la commercialisation de la sexualité, y compris des sexualités minoritaires, tout en faisant appel à l'esprit de Kollontai. La pandémie de 2020 a montré à quel point l'attitude de larges groupes pouvait être conditionnée par la peur de mourir, écrasant la crainte de perdre sa liberté, d'être privé de la richesse des rencontres sociales ou d'être plombé par une liberté surveillée, une ascèse carcérale sans jouissance.

42 Le pôle érotique peut apparaitre aussi dans la réception de pratiques étranges, étrangères, dans l'échange d'idées qui provoquent la surprise ou la joie, dans l'acceptation d'un don ou de l'imprévu. L'apparence étrange, ou l'originalité, définissent d'ailleurs le mot anglais queer au début du 20ème siècle, sans le limiter à une pratique sexuelle particulière ${ }^{98}$. Au vu de la grande difficulté de la gauche en France d'importer des pratiques, des paroles ou des écrits qui lui échappent au début, cela sans vouloir les posséder ou les maitriser, l'idée que le pôle érotique peut encore se déployer se précise. Freud pointe l'hypothèse parfaite - donc irréalisable en pratique - d'un genre de personnalité qui se tiendrait en équilibre sur une pointe, dans la convergences des qualités de trois pôles, frôlant l'harmonie. Comme ce n'est pas vraiment atteignable, surtout dans une société cassée, violente, marquée par l'intensification de la lutte des classes, je me contente ici de nommer un manque érotique. En ce sens, il s'agit d'ouvrir la critique. Freud remarque que la dérive pathologique la plus probable du pôle érotique est l'hystérie, qui sort de ses gonds. Approcher ce phénomène aurait l'avantage de prendre au sérieux l'hystérie masculine, de contrer le cynisme morbide qui veut voir périr le féminisme socialiste, qui accepte que des réfugiés se noient en mer ou qui se reconnait dans les vielles ficelles de l'anticommunisme de droite. La droite internationale veut bien normaliser l'hystérie permanente de la communication du président D. Trump, mais elle ne veut pas admettre l'expression de l'indignation, face aux abus qui visent les femmes, les noirs, les immigrés, les juifs, les travailleurs, les indiens, les lanceurs d'alerte, les socialistes, les antifascistes, les scientifiques. Le cynisme et le refus de la sensibilité caractérisent bien la personnalité autoritaire, 
comme l'a montré Adorno. Tandis que Marcuse avait salué la potentialité démocratique de la nouvelle gauche, favorisant la sensibilité en rapport à l'art, la musique, la culture populaire, la liberté sexuelle, le vivant et l'écologie. Les jeunes générations semblent explorer cet héritage, cet accord secret avec les générations anciennes. Sans verser dans l'hystérie (masculine ou féminine), le pôle érotique peut stimuler une capacité d'indignation qui s'est exprimée matériellement dans la puissance politique du mouvement des Indignados, chez les zapatistes, les lycéens écolos, les Gilets jaunes, les grèves sauvages, le soulèvement de 2020 à l'encontre de meurtres policiers, et de nouvelles pratiques artistiques qui échappent au marché. Les manifestations mondiales, parties de la violence à l'encontre des afro-amércains et des Noirs, ont rapidement pris la tournure d'une créolisation (Edouard Glissant), où toutes les oppositions et cultures qui se manifestent à l'encontre de l'Etat autoritaire peuvent se rencontrer, rappelant en ce sens l'heureuse rencontre Marcuse-Davis-Adorno.

Le manque d'une gauche érotique se manifeste cependant, en France, dans la réticence aux langues étrangères, à la polyphonie, à la créolisation, alors que traductions et éditions sont handicapées, pour évoquer un domaine littéraire et intellectuel. Rouler des pelles littéraires n'a jamais été une qualité de la maison Gallimard, qui a édité la NRF sous l'occupation, après avoir refusé de publier un seul texte de Benjamin. Le nationalisme, pétainiste ou lepéniste, a toujours cherché la mort et Thanatos, dans une obsession jamais démentie. La persistance de traditions hermétiques dans la culture nationale donne un indice de l'enjeu actuel; le nouvel internationalisme viendra avec une ouverture érotique étendue. A Paris, Henri Heine avait nommé cet élan avec poésie : la révolution mondiale ${ }^{99}$.

\section{NOTES}

1. R. Graves, Griechische Mythologie, Rowohlt, 1960, p.48-52.

2. S. Freud, Deuil et mélancolie (1915), Payot, 2016.

3. M. Brumlik, Masse et fascisme, in Variations n.8, issu de : Brumlik, Sigmund Freud - Denker des 20. Jahrhunderts, Beltz, 2006.

4. S. Freud, "Les voies nouvelles de la thérapeutique psychanalytique" in La Technique psychanalytique, PUF, 1981, p. 140-141.

5. S.Freud, Ma vie et la psychanalyse, in : Oeuvres complètes, PUF, 1992, p. 119.

6. S. Freud, Massenpsychologie und Ich-Analyse, Fischer, 1993 (une traduction française évoque la foule, à contre-emploi de l'argumentation de Freud).

7. Lettre de Freud à Eitingon du 25 octobre 1917, cité d'après Peter Gay, Freud, une vie, Fayard, 2011.

8. S. Freud, Die Kalendereinträge von 1916-18, Stromfeld Verlag, 2016.

9. W. Reich, La révolution sexuelle, Christian Bourgeois, 1993 (voir notre traduction revue à partir du texte original de 1937, La révolution sexuelle, in Variations N.23); H. Dahmer, Libido und Gesellschaft. Studien über Freud und die Freudsche Linke. Suhrkamp, 1973 (Münster, 2013); H. Marcuse, Eros et civilisation, Minuit, 1963; F. Gabarron-Garcia, L'héritage politique de la psychanalyse, éditions La Lenteur, 2018. 
10. Le terme Massenpsychologie est utilisé par G. Lukacs, Histoire et conscience de classe, Minuit, 1971. Avec Korsch, Lukacs anime le volet "études dialectiques" du premier séminaire de l'Institut de Francfort en mai 1923.

11. A. Breton, Manifeste du surréalisme (1924), Gallimard. Breton a travaillé dans un centre psychiatrique, avant de rencontrer Freud à Vienne, en 1919, au moment de la révolution des conseils.

12. J.M. Monnoyer, introduction à : Walter Benjamin, Ecrits français, Gallimard, 1991, p.33.

13. W. Benjamin, Paris, Capitale du 19ème siècle, Le Cerf, 2009; Lettre de Benjamin à Adorno, du 10/6/1935, depuis Paris, Correspondance, Gallimard, 2006, p.113.

14. W. Benjamin, Linke Melancholie. Zu Erich Kästners neuem Gedichtbuch (1931), Projekt Gutenberg (tous droits réservés); W. Benjamin, Mélancolie de gauche (1931), revue Variations n.23, Editions critiques / Open edition, 2020, tous droits réservés.

15. E. Kästner, Selbstmord im Familienbad in : Das Erich Kästner Lesebuch, Diogenes, 1989 (nous traduisons).

16. R. Keucheyan, Hemisphère gauche, La Découverte, 2016, p.34.

17. Lettre de W. Benjamin à Gretel Adorno du 20 juillet 1938 in : Benjamin, Briefe, III, Suhrkamp, 1997; voir J.M. Monnoyer, introduction à : W. Benjamin, Ecrits français, Gallimard, 1991, p.17.

18. K.S. Larsson, The dissemination of false data through inadequate citation, Journal of Internal Medicine n. 238-5, 1995, pp. 445-450.

19. Traverso, p.32. Voir P. Anderson, Sur le marxisme occidental, Maspero 1972 (et la postface de l'autre de 1974 où il révise complètement sa position).

20. P. Anderson, Sur le marxisme occidental, Maspero, 1976, p.149.

21. G. Lukacs, Grand Hôtel Abgrund, 1933, trad. fr. : http://amisgeorglukacs.org/2017/07/georglukacs-grand-hotel-de-l-abime-1933.html

22. G.Lukacs, préface allemande à la Théorie der Novelle, Luchterhand, 1951.

23. Adorno, Minima moralia, Suhrkamp, 1951.

24. Z. Sternhell, Ni droite ni gauche. L'idéologie fasciste en France, Gallimard, 2012, p.110. Etant donné que la maison Gallimard a elle-même publié ce constat d'un historien, il peut être considéré comme parfaitement fiable.

25. W. Benjamin, Mélancolie de gauche, 1931, Variations n.23, 2020.

26. E. Traverso, Mélancolie de gauche, La Découverte/Poche, 2016/2018.

27. E. Traverso, op.cit., p.121.

28. W. Benjamin, Zur Lage der russischen Filmkunst. Erwiderung an Oskar H. Schmitz, in : Die literarische Welt n.10,11/3/1927, réedition in Dieter Prokop, dir., Materialien zur Theorie des Films, ed. Hanser, 1971 (voir aussi : Benjamin, Discussion du cinéma russe in: Les Cahiers du Cinéma n.226, 1/1971) ; Benjamin, Das Kunstwerk im Zeitalter seiner technischen Reproduzierbarkeit (1936), Reclam, 2013.

29. Concernant la technique de montage d'Eisenstein repris par Trotsky, voir I.Deutscher, Trotsky, vol. 5 (Le prophète hors la loi 1), Juliard, 1980, pp.318-19. Voir Trotsky, Geschichte der russischen Revolution (1930), Fischer, Verlag, 1989. Traverso assimile ce livre de Trotsky à un film hagiographique d'Eisenstein (Octobre, 1928) qui subit alors la censure et la terreur stalinienne (Traverso, L'histoire comme champ de bataille, La découverte, 2011, p.59).

30. A. Kluge, Nachrichten aus der ideologischen Antike. Marx-Eisenstein, Edition Suhrkamp (DVD), 2012.

31. K. Marx, K. Marx, Critique du programme de Gotha (1975), GEME/ES, 2008.

32. W. Benjamin, Sur le concept d'histoire (1940), Payot, 2017.

33. H. Arendt, Die verborgene Tradition, Suhrkamp, 2016.

34. H. Arendt, Der Zionismus aus heutiger Sicht in : Die verborgene Tradition, Surkamp, 2016, pp. 127-166. 
35. H. Arendt, Aufklärung und Judenfrage, in : op.cit., pp.108-126. Voir la contre-proposition : W. Benjamin, Ecrits français, Gallimard, 1991, pp.347-50

36. H. Arendt, Juden in der Welt von Gestern, in : op.cit., pp.74-87.

37. W. Benjamin, Kollektive Impfung: Mickey Mouse et Chaplin, in: Das Kunstwerk im Zeitalter seiner technischen Reproduzierbarkeit, Reclam 2013, p.61.

38. W. Benjamin, Sur le concept d'histoire, Payot, 2017 : thèse VI (nous traduisons : Benjamin, Über den Begriff der Geschichte in : Sprache und Geschichte, Reclam, 2000, p.144).

39. W. Benjamin, Über den Begriff der Geschichte (1940), thèse II, Reclam, 2000, p.141.

40. M. Horkheimer, Théorie traditionnelle et théorie critique (1931), Gallimard, 1974.

41. Theodor W. Adorno, Dissonanzen, Suhrkamp, 2003, p.132.

42. Adorno/Benjamin, Correspondance 1928-1940, Gallimard, 2006.

43. Heidegger zertrümmern, thème d'un séminaire de recherche que Benjamin projetait d'organiser à Paris avec Brecht durant l'exil, mais qui a été empêché par les évènements historiques. Voir W. Benjamin, Briefe III, Suhrkamp, p.522.

44. Walter Benjamin, Paris, Capitale du 19ème siècle (Le livre des passages), Cerf, 2009, pp. 477-94.

45. (Tiedemann), Zeugnisse sur Entstehungsgeschichte, in : W. Benjamin, Das Passagenwerk, 2, Suhrkamp, 1982, pp.1081-1205.

46. Adorno, Charakteristik Walter Benjamins, in: Kulturkritik und Gesellschaft I, Suhrkamp, 1997, p. 241.

47. Th. W. Adorno, Ontologie und Dialektik (1961), Suhrkamp, 2001; Adorno, Dialectique négative (1966), Payot, 2003.

48. Adorno, Dialectique négative (1966), Payot, 2003.

49. P. Trawny, Was ist deutsch?, M\&S, 2016.

50. A. Schmieder, Linke Melancholie in : Socialnet.de, 8 mai 2020.

51. Lettre d'Adorno à Benjamin du 5/6/1935, in Adorno/Benjamin, Correspondance, Gallimard, 2006, p.105-107.

52. W. Benjamin, Ecrits français, Gallimard, 1991, pp.347-50.

53. Traverso, op.cit., pp.17-21.

54. D. Bensaïd, Marx l'intempestif, Fayard, 1995, p.101.

55. Traverso, op.cit., p.2.

56. Traverso, p.9 : "La fin du communisme a brisé cette dialectique entre passé et futur, et l'éclipse des utopies qui accompagne notre époque présentiste a conduit à la quasi extinction de la mémoire marxiste. La tension entre passé et futur est devenu une 'dialectique négative', mutilée."

57. Adorno, Minima moralia. Reflexionen aus dem beschädigten Leben, Suhrkamp, 1951. Les réflexions sont menées à partir d'une vie endommagée, mais elle ne pourraient pas se faire si la vie était définitivement mutilée, tel un membre perdu pendant la guerre.

58. Adorno/Horkheimer, Towards a new Manifesto (1956), Verso Books, 2012.

59. H. Marcuse, Le marxisme soviétique, Gallimard, 1959.

60. Traverso, op.cit., pp.185-203.

61. D. Bensaid, Stratégie et parti, éditions la Brèche-PEC, 1987.

62. (D. Bensaid et alli) A la gauche du possible. Ce que propose la Ligue communiste révolutionnaire, éditions La Brèche-PEC, 1991, p.29.

63. E. Traverso, op.cit. p.14.

64. Arruza/Bhattacharya/Fraser, Féminisme pour les 99\%, La Découverte, 2019.

65. K. Marx : "Je ne suis pas marxiste!", cité d'après F.Engels, en français dans le texte, MEW 37, Dietz, p.463.

66. M. Löwy, Avertisseur d'incendie, PUF, 2003. Voir Traverso, op.cit., p. 8. 
67. D. Bensaïd, Le pari mélancolique, Fayard, 2003.

68. O. Negt, Überlebensglück, Steidl, 2018.

69. Traverso, op.cit. p.189. Ces références n'apparaissent par exemple nulle part dans le livreprogramme de D. Bensaïd, Marx l'intempestif, Fayard, 1995.

70. M. Foucault, Structuralisme et poststructuralisme (entretien avec G. Raulet), in : Dits et écrits, vol. 4, Gallimard, p.73.

71. D. Bensaïd, Les intellectuels du PCF dos au stalinisme, in : J.-M. Vincent, dir., Contre Althusser, 10/18, p.295;D. Bensaïd, Marx l'intempestif, Fayard, 1995; voir A. Neumann, Chorégraphier le chaos : Hommage à Daniel Bensäid dans le cadre des colloques organisés pour les 50 ans de l'Université Vincennes-Paris 8 (Eric Lecerf et alli), 2018.

72. W. Benjamin, Mélancolie de gauche (1931), revue Variations n.23, Editions critiques / Open edition, 2020.

73. P. Bourdieu, La Distinction, Minuit, 1979.

74. Pour sa part Traverso détient la position d'un professeur de Cornell, une école supérieure privée US qui porte le nom du fondateur du service financier Western Union (aujourd'hui pourvu d'un chiffre d'affaires annuel d'un milliard de dollars US).

75. W. Benjamin, Mélancolie de gauche, op.cit.

76. Pour une discussion plus ample de la posture poststructuraliste, voir R. Garcia, Le désert de la critique, L'échappée, 2015 et A. Neumann, La révolution et nous - 230 ans de Théorie critique (1791-2021), Editions critiques, à paraitre en février 2021.

77. L. Althusser, Pour Marx, Maspero, 1965; E. Balibar, E. Wallerstein, Race, classe, nation (1988), La Découverte, 2018.

78. S. Hall, Signification, Representation, Ideology :Althusser and the poststructuralist debates, CSCM - Critical studies in mass communication, Vol.2 n.2, 6/1985, p.91-140 (traduction française in Raisons pratiques n.48, 2012).

79. Traverso, op.cit., p.177.

80. A. Davis, Femmes, classe, race (1980), Antoine Fouque, 2020. Position rappelée par Arruza/ Bhattacharya/Fraser, Féminisme pour les 99\%, La Découverte, 2019.

81. J. Holloway, F. Matamoros, S. Tischler (dir.), Negativity and revolution : Adorno and political activism, Pluto Press, 2008.

82. M. Acikgöz, Die Permanenz der kritischen Theorie, W. Dampfboot, 2014.

83. J. Rancière, La leçon d'Althusser (1974), La Fabrique, 2012; E.P. Thompson, Misère de la théorie. Contre Althusser et le marxisme anti-humaniste, L'échappée, 2014; C. Castoriadis, Les crises d'Althusser. De la langue de bois à la langue de caoutchouc, Libre, N.4, 1978, pp. 239-54; J.M. Vincent (dir.), Contre Althusser, 10/18, 1974; D. Bensäid (dir.), Contre Althusser - Pour Marx, La Passion, 1999.

84. J. Derrida, Force de loi, Gallilée, 1994.

85. J. Derrida, Spectres de Marx, Gallilée, 1993, p.11.

86. J. Butler, Freud et la mélancolie du genre, in : Trouble dans le genre, La Découverte, 2000.

87. M. Foucault, Structuralisme et poststructuralisme (entretien avec G. Raulet), in : Dits et écrits, vol. 4, Gallimard, p.73. Ici, Foucault précise que le terme poststructuralisme ne veut rien dire.

88. The Philosophy and Literature Bad Writing Award, attribué au texte de J. Butler, Further Reflections on the Conversations of our Time, Diacritics, Vol.27, 1997.

89. L. Althusser, Idéologie et appareils idéologiques d'État in : Positions (1964-1975), Éditions sociales, Paris, 1976, p. 172.

90. J. Butler, Deux lectures du jeune Marx, Editions Sociales, 2019.

91. L. Althusser, L'avenir dure longtemps, Stock, 1992.

92. E. Traverso, op.cit., p.7.

93. W. Benjamin, Mélancolie de gauche (1931), Variations n.23.

94. A. Kollontai, Marxisme et révolution sexuelle, Maspero, 1973. 
95. S. Freud, Des types libidinaux, 1931 in : Oeuvres 1931-36, PUF.

96. L. Althusser, L'avenir dure longtemps, Stock, 1992; A. Gorz, Le traitre (1959), Gallimard, 2005; J.P. Sartre, Les mots, Gallimard, 1990.

97. A. Kollontai, Marxisme et révolution sexuelle, Maspéro, 1973; H. Marcuse, Eros et civilisation, Ed. de Minuit, 1972; D. Guérin, Homosexualité et révolution, Le front social, 1983; V. Despentes, King Kong théorie, Grasset, 2003.

98. Cassels New Dictionnary, F\&W, 1906, p.367.

99. H.Heine, Lutetia, XLVI, Paris, 12/7/1842, Reclam, 1920.

INDEX

Mots-clés : Théorie critique, Ecole de Francfort, Mélancolie de gauche, Walter Benjamin, Enzo Traverso

\section{AUTEUR}

\section{ALEXANDER NEUMANN}

Professeur des Universités, Paris 8 